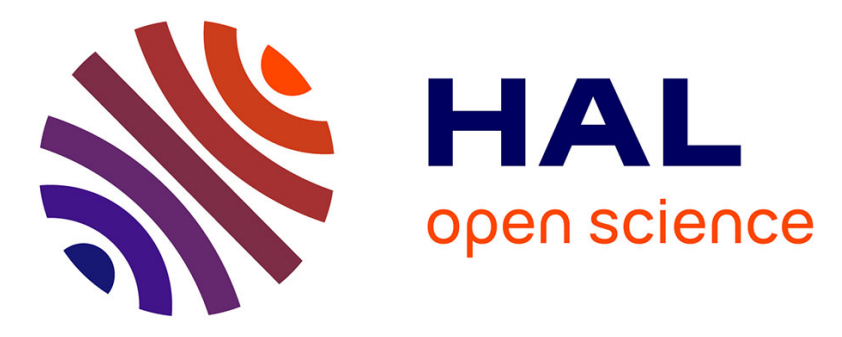

\title{
IKZF1 alterations predict poor prognosis in adult and pediatric T-ALL
}

\author{
Mathieu Simonin, Ludovic Lhermitte, Marie-Emilie Dourthe, Etienne \\ Lengline, Carlos Graux, Nathalie Grardel, Jean-Michel Cayuela, Isabelle \\ Arnoux, Virginie Gandemer, Norbert Ifrah, et al.
}

\section{To cite this version:}

Mathieu Simonin, Ludovic Lhermitte, Marie-Emilie Dourthe, Etienne Lengline, Carlos Graux, et al.. IKZF1 alterations predict poor prognosis in adult and pediatric T-ALL. Blood, 2021, 137 (12), pp.1690-1694. 10.1182/blood.2020007959 . hal-03122574

\section{HAL Id: hal-03122574 \\ https://hal.science/hal-03122574}

Submitted on 5 Feb 2021

HAL is a multi-disciplinary open access archive for the deposit and dissemination of scientific research documents, whether they are published or not. The documents may come from teaching and research institutions in France or abroad, or from public or private research centers.
L'archive ouverte pluridisciplinaire HAL, est destinée au dépôt et à la diffusion de documents scientifiques de niveau recherche, publiés ou non, émanant des établissements d'enseignement et de recherche français ou étrangers, des laboratoires publics ou privés. 


\title{
IKZF1 alterations predict poor prognosis in adult and pediatric T-ALL
}

\author{
Tracking no: BLD-2020-007959R2
}

Mathieu Simonin (Armand Trousseau Hospital, AP-HP and Sorbonne University, France) Ludovic Lhermitte (Assistance Publique-Hôpitaux de Paris (AP-HP), Hôpital Necker Enfants-Malades, Paris, France, France) Marie-Emilie Dourthe (Hôpital Necker enfants malades, France) Etienne Lengline (Hopital Saint-Louis APHP, France) Carlos Graux (CHU UCL Namur - Godinne, Belgium) nathalie grardel (CHRU de lille, France) Jean-Michel CAYUELA (University Hospital Saint-Louis, APHP and Paris DIDEROT, France) Isabelle Arnoux (La Timone Hospital, France) Virginie GANDEMER (CNRS UMR6061, France) Norbert Ifrah (Centre Hospitalier Universitaire Angers, France) Hervé Dombret (Hopital Saint-Louis, France) André Baruchel (Hôpital Robert Debré (AP-HP) and Université de Paris, France) Elizabeth Macintyre (INSERM U1151, Université de Paris, Institut Necker-Enfants Malades, Assistance Publique-Hopitaux de Paris, France) Arnaud Petit (Hopital Armand Trousseau, Assistance Publique -Hopitaux De Paris, Sorbonne Université, France) Nicolas Boissel (Hopital Saint-Louis, AP-HP, France) Vahid Asnafi (Laboratory of Onco-Hematology, Assistance PubliqueHopitaux De Paris (AP-HP), Hopital Necker Enfants-Malades, France)

\section{Abstract:}

Conflict of interest: No COI declared

COI notes:

Preprint server: No;

Author contributions and disclosures: N.B and V.A and M.S conceived the study and oversaw the project; M.S, ME.D, L.L, E.L, C.G, N.G, JM.C, I.A, V.G, N.I, H.D, A.B, A.P, N.B provided study materials or patients; M.S, L.L, E.M and V.A performed molecular analyses; M.S, L.L, V.A. collected and assembled data; N.B and M.S performed statistical analysis; M.S, L.L, V.A, N.B analyzed and interpreted data; M.S, N.B, E.M, V.A wrote the manuscript; all authors approved the manuscript.

Non-author contributions and disclosures: No;

Agreement to Share Publication-Related Data and Data Sharing Statement: emails to the corresponding author

Clinical trial registration information (if any): 215 adult patients aged from 16-59 years, were included in the GRAALL03/05 trials which were registered at clinicaltrials.gOv (GRAALL-2003, \#NCT00222027; GRAALL-2005, \#NCT00327678). About 261 pediatric patients aged from 1-19 years, were treated in 10 French pediatric hematology departments, members of the FRALLE study group, according to the FRALLE 2000 T guidelines. 


\section{IKZF1 alterations predict poor prognosis in adult and pediatric T-ALL}

Mathieu Simonin $^{1,2}$, Ludovic Lhermitte ${ }^{1}$, Marie-Emilie Dourthe ${ }^{1,3}$, Etienne Lengliné ${ }^{4}$, Carlos Graux ${ }^{5}$, Nathalie Grardel ${ }^{6}$, Jean-Michel Cayuela ${ }^{7}$, Isabelle Arnoux ${ }^{8}$, Virginie Gandemer ${ }^{9}$, Norbert Ifrah ${ }^{10}$, Hervé Dombret $^{4}$, André Baruchel ${ }^{3}$, Elizabeth Macintyre ${ }^{1}$, Arnaud Petit ${ }^{2}$, Nicolas Boissel ${ }^{4 *}$, Vahid Asnafi ${ }^{{ }^{*}}$

Affiliations:

${ }^{1}$ Université de Paris (Descartes), Institut Necker-Enfants Malades (INEM), Institut national de la santé et de la recherche médicale (Inserm) U1151, and Laboratory of Onco-Hematology, Assistance

Publique-Hôpitaux de Paris, Hôpital Necker Enfants-Malades, Paris, France

${ }^{2}$ Department of Pediatric Hematology and Oncology, Assistance Publique-Hôpitaux de Paris (AP-HP), GH HUEP, Armand Trousseau Hospital, Paris, France and Sorbonne Universités, UPMC Univ Paris 06, UMRS 938, CDR Saint-Antoine, GRC n07, GRC MyPAC, Paris, France

${ }^{3}$ Department of Pediatric Hematology and Immunology, Robert Debré Hospital, AP-HP and University Paris Diderot, Paris, France

${ }^{4}$ Université Paris Diderot, Institut Universitaire d'Hématologie, EA-3518, Assistance PubliqueHôpitaux de Paris, University Hospital Saint-Louis, Paris, France.

${ }^{5}$ Department of Hematology, Université catholique de Louvain, CHU UCL Namur - site Godinne, Yvoir, Belgium

${ }^{6}$ Laboratory of Hematology, CHRU Lille, Lille, France and U1172, INSERM, LILLE, France

7'Laboratory of Hematology and EA 3518 University Hospital Saint-Louis, AP-HP and Université de Paris

${ }^{8}$ Hematology Laboratory, Marseille University Hospital Timone, Marseille, France

${ }^{9}$ Department of Pediatric Hematology and Oncology, University Hospital of Rennes, Rennes, France

${ }^{10}$ PRES LUNAM, CHU Angers service des Maladies du Sang et INSERM U 892, Angers, France,

*: Co-coresponding authors

Short title: IKZF1 alterations predict poor outcome in T-ALL

Corresponding author:

Vahid Asnafi, Laboratory of Onco-Hematology, Tour Pasteur 2eme etage, Necker Enfants Malades Hospital, 149 rue de Sevres, 75015 Paris, France.

E-mail: vahid.asnafi@aphp.fr ; Phone: +33 144.49.49.33

Nicolas Boissel, Hôpital Saint-Louis,

Service d'Hématologie Adulte, 1 avenue Claude Vellefaux 75010 Paris, France

E-mail: nicolas.boissel@aphp.fr ; Phone: (+33) 142499643 ; Fax: (+33) 142494010 
T-cell acute lymphoblastic leukemia (T-ALL) and T-cell lymphoblastic lymphoma (T-LBL) are aggressive neoplasms which result from the proliferation of T-lymphoid progenitors blocked at thymic stages of differentiation. They account for $15 \%$ and $25 \%$ of pediatric and adult ALLs, respectively. T-ALL/LBL are associated with a wide range of acquired genetic abnormalities that contribute to developmental arrest and abnormal proliferation. ${ }^{1,2}$ Although intensive treatment protocols have markedly improved the outcomes of children with T-ALL, cure rates remain below $60 \%$ for adults and $85 \%$ for children. ${ }^{3-5}$ The prognosis is particularly poor in relapsing patients, highlighting an urgent need for risk stratification factors at diagnosis. ${ }^{6,7}$

The IKAROS transcription factor (encoded by the IKZF1 gene on chromosome 7p12.2) is a member of the zinc finger family of DNA-binding proteins which acts as a critical regulator of hematopoiesis and lymphoid differentiation. ${ }^{8}$ IKZF1 is recurrently affected by various genetic alteration in B-cell acute lymphoblastic leukemia (B-ALL). Genomic alterations in IKZF1 are found in about $15 \%$ of childhood and $40 \%$ of adult B-ALL cases, with a higher incidence in poor prognosis cases, including $B C R-A B L 1$ (70\%) or BCR-ABL1-like (40\%) B-ALL., ${ }^{9,10}$ Of note, IKZF1 alteration consistently demonstrated its poor prognostic impact in B-ALL and clinical trials increasingly integrate IKZF1 gene status in risk stratification algorithms. ${ }^{11,12}$

In contrast, both the incidence and prognostic influence of IKZF1 alterations in T-ALL/LBL are poorly characterized. ${ }^{13}$ To specify the role of IKZF1 alterations in T-ALL/LBL we conducted a comprehensive analysis using pan-exon deep sequencing of 1260 adult and pediatric T-ALL/LBL patients (Flow chart, supplemental Figure 2) including 980 T-ALL and 280 T-LBL. Diagnostic DNA samples were analyzed using an 80-gene pan-exon capture-panel (details included in supplemental Methods). IKZF1 Alt screening was performed by computational approaches previously described for the detection of copy number variants (CNVs) from next-generation sequencing data. ${ }^{14}$ IKZF1 deletions were confirmed with Multiplex ligation-dependent probe amplification (MLPA) analysis and/or microarray based comparative genomic hybridization (CGHarray). Patient protocols and 
clinical trials $3,15,16$, immunophenotypic and molecular characterization of T-ALL and T-LBL samples, minimal residual disease (MRD) assessment, gene mutation screening, array CGH, MLPA, statistical analysis, and additional details are included in supplemental Methods.

IKZF1 mutations were identified in 42 cases including 33/980 (3.4\%) and 9/280 (3.2\%) of TALL and T-LBL respectively (Figure $1 \mathrm{~A})$. The majority of mutations were missense $(24 / 42,60 \%)$ within a mutational hotspot in exon 5 affecting amino acid p.N159 (N159S/T) located in the DNA-binding domain. Interestingly, this mutation was recently described in a new combined immunodeficiency syndrome with potential risk of T-ALL predisposition. ${ }^{17,18}$ We also detected frameshift or nonsense mutations $(17 / 42,40 \%)$ affecting exons $3-8$ and predicted to truncate the protein before the Cterminal dimerization domain resulting in haploinsufficiency. IKZF1 deletions were detected in 40 cases (3\%) including $37 / 980(3.8 \%)$ and $3 / 280(1.5 \%)$ in T-ALL and T-LBL respectively (Figure 1B). All were confirmed by MLPA and/or CGH-array (supplemental Figure 2 and supplemental Figure 3A-B). Of note, most cases (30/40, 75\%) harbored pan-genic deletions (exons 1-8) leading to haploinsufficiency and only $10 \%(4 / 40)$ intragenic deletions (exons $4-7$ ), predicted to induce a dominant-negative effect, were observed. This suggests that, in contrast to BCP-ALL ${ }^{12}$, the main consequences of IKZF1 deletions in T-ALL would be haplo-insufficiency rather than a dominantnegative effect. IKZF1 deletions and mutations were mutually exclusive and no biallelic inactivation of IKZF1 was observed, suggesting that residual IKZF1 activity may be required for T-lineage leukemogenesis. Overall, IKZF1 ${ }^{\mathrm{Alt}}$ was identified in 82/1260 (6.5\%) T-ALL/LBL (7.1\% T-ALL and 4.3\% TLBL) and were more frequent within adult T-ALL/LBL as compared to pediatric cases (54/699 adult TALL/LBL as compared to $28 / 561$ pediatric cases, $p=0.05$, supplemental Figure 2 ). The oncoplot highlighting the main co-mutations observed and mutations within individual IKZF1 ${ }^{\text {Alt }}$ cases are reported in supplemental table S3 and Figure S4.

We then investigated the clinical characteristics linked to IKZF1 $1^{A / t}$ in a subset of 476 patients, including 215 adults enrolled in the GRAALL-2003/05 trials and 261 children enrolled in the FRALLE- 
2000 trial (Table1) and supplemental methods (GRAALL-2003, \#NCT00222027; GRAALL-2005, \#NCT00327678). Diagnostic peripheral blood or bone marrow samples from 1258 adults and children with T-ALL or LBL-T were analyzed after informed consent was obtained at diagnosis according to the Declaration of Helsinki. The incidence of IKZF1Alt in this cohort was 5.5\% (26/476), including 16 deletions and 10 mutations. IKZF1 ${ }^{A / t}$ were observed in $7 \%$ of adults and $4.2 \%$ of children $(P=0.2)$ with a median age slightly higher in $I K Z F 1^{\text {Alt }}$ cases $\left(23.5\right.$ years vs. 15.2 years, $\mathrm{P}=0.1$ ). IKZF1 ${ }^{\text {Alt }}$ was associated with an immature immunophenotype (47\% vs. $20 \%, P=0.02)$. In line with this, IKZF1 $1^{A / t}$ correlated positively with abnormalities known to be associated with an immature phenotype, including $\mathrm{K} / \mathrm{N}-\mathrm{RAS}$ mutations ( $31 \%$ vs. $9 \%, \mathrm{P}=0.003)$, , $E Z H 2(5 / 26,20 \%$ vs $27 / 450,6 \%, P=0.02)$, $\operatorname{ASXL1}(4 / 26,15 \%$ vs $15 / 450,3 \%, P=0.02), \operatorname{ETV} 6(4 / 26,15 \%$ vs $13 / 450,3 \%, P=0.01)$ and DNMT3A $(5 / 26,20 \%$ vs $17 / 450,4 \%, P=0.045)$ mutations (Figure $1 C$ ). Conversely, IKZF1 ${ }^{A / t}$ were virtually exclusive with SIL-TAL1+ and PTEN altered cases known to be associated with a mature TCR $\alpha \beta$ lineage.

In BCP-ALL, IKZF1 ${ }^{A / t}$ are enriched in the high-risk subgroup of $P h^{+} \mathrm{BCP}-\mathrm{ALL}$ and the recently characterized by the presence of a gene expression profile (GEP) similar to $\mathrm{Ph}^{+} \mathrm{ALL}$ but lacking the canonical $B C R-A B L 1$ fusion, therefore named $B C R-A B L 1$-like or $P h^{+}$-like ALL., ${ }^{9,19}$ Importantly, $P h^{+}$-like signature is virtually absent in T-ALL ${ }^{20}$. In contrast, here we observed that $I K Z F 1^{A / t}$ are associated with epigenetic mutations/deletions.

Interestingly, IKZF1-deficient mice develop T-cell malignancy with high penetrance highlighting the suppressor function for IKAROS in T-cell lineage. ${ }^{21}$ Furthermore, in murine T-cell leukemogenesis IKAROS directly cooperates with NOTCH1 activation to promote leukemia. ${ }^{22,23}$ This crosstalk between NOTCH1 and IKAROS could explain the discrepancy observed concerning the pattern of IKZF1 ${ }^{A / t}$ in T-ALL vs $P h^{+}$-like BCP-ALL and led us to suspect a specific oncogenic mechanism associated with IKZF1 $1^{A / t}$ in T-ALL requiring further investigations. 


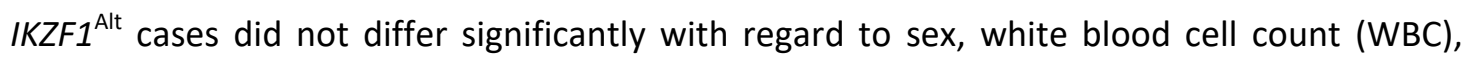
central nervous system involvement and prednisone response (Table1). Although $I K Z F 1^{A / t}$ did not impact complete remission rate, patients with $I K Z F 1^{A / t}$ were more likely to have a positive postinduction MRD $\left(10^{-4}\right.$ threshold, $67 \%$ vs $\left.34 \% ; P=0.01\right)$. Patients with $I K Z F 1^{A l t}$ had an inferior outcome compared to $I K Z F 1^{G L}$, with a higher cumulative incidence of relapse $(5 y-C I R: 50 \%$ vs. $28 \%$; specific hazard ratio $2.12,95 \% \mathrm{CI}[1.17-3.86]$ ) and a shorter overall survival (5y-OS: $37 \%$ vs. $73 \%$; hazard ratio: 2.94, 95\% CI[1.74-4.96]) (Table1 and Figure 1D-E). This prognostic impact was observed in both pediatric and adult cohorts (supplemental Figures S5A-D). Of note the 10 IKZF1 mutated and 16 IKZF1 deleted cases demonstrated comparable clinico-biological features and were associated with worse prognosis when compared to $I K Z F 1^{G L}$ cases (supplemental table S4 and figure S6). In multivariate analysis considering variables associated with $\mathrm{OS}$ in univariate analyses as covariates, IKZF1 ${ }^{\text {Alt }}$ remained significantly associated with a shorter OS (Table 1), even after inclusion of post-induction MRD in the model (supplemental table S5). It is noteworthy that the prognostic impact of IKZF1 ${ }^{A l t}$ status was also observed after adjustment on the 4-gene NOTCH1/FBXW7/RAS/PTEN classifier and post-induction MRD which identified poor prognosis patients in both GRAALL and FRALLE trials. ${ }^{3,4}$

In conclusion, we describe IKZF1 ${ }^{\text {Alt }}$ among 1260 children and adults with immature T-ALL/LBL and define for the first time its frequency and, importantly, its poor outcome in T-ALL in multivariate models. IKZF1 ${ }^{\mathrm{Alt}}$ should be considered as a significant prognosis marker in addition to MRD and the 4gene oncogenetic classifier to predict poor outcomes T-ALL. 


\section{Acknowledgements}

The authors would like to thank all participants in the GRAALL-2003 and GRAALL-2005 study groups, the SFCE and the investigators of the 16 SFCE centers involved in collection and provision of data and patient samples, and V. Lheritier for collection of clinical data. The GRAALL was supported by grants P0200701 and P030425/AOM03081 from the Programme Hospitalier de Recherche Clinique, Ministère de l'Emploi et de la Solidarité, France and the Swiss Federal Government in Switzerland. Samples were collected and processed by the AP-HP "Direction de Recherche Clinique" Tumor Bank at Necker-Enfants Malades. MS was supported by "Soutien pour la formation à la recherche translationnelle en cancérologie dans le cadre du Plan cancer 2009-2013 ». This work was supported by grants to Necker laboratory from the "Association Laurette Fugain", Association pour la Recherche contre le Cancer (Equipe labellisée), Institut National du Cancer PRT-K 18-071 and the Fédération Leucémie espoir and Horizon Hemato.

\section{Authorship contributions}

N.B and V.A and M.S conceived the study and oversaw the project; M.S, ME.D, L.L, E.L, C.G, N.G, JM.C, I.A, V.G, N.I, H.D, A.B, A.P, N.B provided study materials or patients; M.S, L.L, E.M and V.A performed molecular analyses; M.S, L.L, V.A. collected and assembled data; N.B and M.S performed statistical analysis; M.S, L.L, V.A, N.B analyzed and interpreted data; M.S, N.B, E.M, V.A wrote the manuscript; all authors approved the manuscript.

\section{Disclosure or Conflicts of Interest}

The authors declare no competing financial interests. 


\section{References}

1. Van Vlierberghe P, Ferrando A. The molecular basis of T cell acute lymphoblastic leukemia. J. Clin. Invest. 2012;122(10):3398-3406.

2. Girardi T, Vicente C, Cools J, De Keersmaecker K. The genetics and molecular biology of T-ALL. Blood. 2017;129(9):1113-1123.

3. Petit A, Trinquand A, Chevret $S$, et al. Oncogenetic mutations combined with MRD improve outcome prediction in pediatric T-cell acute lymphoblastic leukemia. Blood. 2018;131(3):289-300.

4. Trinquand A, Tanguy-Schmidt A, Ben Abdelali R, et al. Toward a NOTCH1/FBXW7/RAS/PTENbased oncogenetic risk classification of adult T-cell acute lymphoblastic leukemia: a Group for Research in Adult Acute Lymphoblastic Leukemia study. J. Clin. Oncol. 2013;31(34):4333-4342.

5. Gökbuget N, Kneba M, Raff T, et al. Adult patients with acute lymphoblastic leukemia and molecular failure display a poor prognosis and are candidates for stem cell transplantation and targeted therapies. Blood. 2012;120(9):1868-1876.

6. Gökbuget N, Stanze D, Beck J, et al. Outcome of relapsed adult lymphoblastic leukemia depends on response to salvage chemotherapy, prognostic factors, and performance of stem cell transplantation. Blood. 2012;120(10):2032-2041.

7. Desjonquères $A$, Chevallier $P$, Thomas $X$, et al. Acute lymphoblastic leukemia relapsing after first-line pediatric-inspired therapy: a retrospective GRAALL study. Blood Cancer J. 2016;6(12):e504.

8. Georgopoulos K, Bigby M, Wang JH, et al. The Ikaros gene is required for the development of all lymphoid lineages. Cell. 1994;79(1):143-156.

9. Mullighan CG, Su X, Zhang J, et al. Deletion of IKZF1 and Prognosis in Acute Lymphoblastic Leukemia. New England Journal of Medicine. 2009;360(5):470-480.

10. Mullighan CG, Goorha S, Radtke I, et al. Genome-wide analysis of genetic alterations in acute lymphoblastic leukaemia. Nature. 2007;446(7137):758-764.

11. Stanulla M, Cavé H, Moorman AV. IKZF1 deletions in pediatric acute lymphoblastic leukemia: still a poor prognostic marker? Blood. 2020;135(4):252-260.

12. Vairy S, Tran TH. IKZF1 alterations in acute lymphoblastic leukemia: The good, the bad and 
the ugly. Blood Rev. 2020;100677.

13. Marçais $A$, Jeannet $R$, Hernandez $L$, et al. Genetic inactivation of Ikaros is a rare event in human T-ALL. Leuk. Res. 2010;34(4):426-429.

14. Nord AS, Lee M, King M-C, Walsh T. Accurate and exact CNV identification from targeted high-throughput sequence data. BMC Genomics. 2011;12:184.

15. Huguet $F$, Leguay $T$, Raffoux E, et al. Pediatric-inspired therapy in adults with Philadelphia chromosome-negative acute lymphoblastic leukemia: the GRAALL-2003 study. J. Clin. Oncol. 2009;27(6):911-918.

16. Huguet $\mathrm{F}$, Chevret $\mathrm{S}$, Leguay $\mathrm{T}$, et al. Intensified Therapy of Acute Lymphoblastic Leukemia in Adults: Report of the Randomized GRAALL-2005 Clinical Trial. J. Clin. Oncol. 2018;36(24):2514-2523.

17. Boutboul D, Kuehn HS, Van de Wyngaert Z, et al. Dominant-negative IKZF1 mutations cause a T, B, and myeloid cell combined immunodeficiency. J. Clin. Invest. 2018;128(7):3071-3087.

18. Yoshida N, Sakaguchi H, Muramatsu H, et al. Germline IKAROS mutation associated with primary immunodeficiency that progressed to T-cell acute lymphoblastic leukemia. Leukemia. 2017;31(5):1221-1223.

19. MI DB, M van S, Rx DM, et al. A Subtype of Childhood Acute Lymphoblastic Leukaemia With Poor Treatment Outcome: A Genome-Wide Classification Study. The Lancet. Oncology. 2009;10(2):.

20. van der Veer A, Waanders $E$, Pieters $R$, et al. Independent prognostic value of BCR-ABL1-like signature and IKZF1 deletion, but not high CRLF2 expression, in children with B-cell precursor ALL. Blood. 2013;122(15):2622-2629.

21. Marke R, van Leeuwen FN, Scheijen B. The many faces of IKZF1 in B-cell precursor acute lymphoblastic leukemia. Haematologica. 2018;103(4):565-574.

22. Mantha S, Ward M, McCafferty J, et al. Activating Notch1 mutations are an early event in Tcell malignancy of Ikaros point mutant Plastic/+ mice. Leukemia Research. 2007;31(3):321-327.

23. Kleinmann E, Geimer Le Lay A-S, Sellars M, Kastner P, Chan S. Ikaros represses the transcriptional response to Notch signaling in T-cell development. Mol. Cell. Biol. 2008;28(24):74657475. 


\section{Table 1:}

Clinico-biological and outcome characteristics of adult and pediatric T-ALLs (GRAALL and FRALLE protocols) according to IKZF1 status.

Abbreviations: T-ALL: T-cell acute lymphoblastic leukemia; WBC, white blood count; CNS, central nervous system; ETP, early thymic precursor; N/F-R/P classifier, NOTCH1/FBXW7-RAS/PTEN classifier as previously described; CR, complete remission; MRD, minimal residual disease; HSCT, hematopoietic stem cell transplantation; CIR, cumulative incidence of relapse; OS, overall survival. MRD1 correspond to MRD evaluation after induction and was performed by allele-specific oligonucleotides polymerase chain reaction. T-cell receptor status and oncogenic were performed as described in supplemental methods.

Univariate and Multivariate analyses of Cumulative Incidence of Relapse and Overall Survival: *continuous variables; **Presence of RAS/PTEN alteration and/or absence of NOTCH1/FBXW7 mutations. Abbreviations: CIR: cumulative incidence of relapse, OS: overall survival, HR: hazard ratio, SHR: specific hazard ratio, $\mathrm{Cl}$ : confidence interval.

\section{Figures legends}

Figure 1: Figure 1A-E: IKZF1 mutational and deletion patterns by patient occurrence.

(A). Gene map describing IKZF1 intragenic mutational patterns by patient occurrence. (B). Gene map describing IKZF1 deletion patterns by patient occurrence. (C). Genetic profiles of IKZF1 ${ }^{\mathrm{Alt}} \mathrm{T}$-ALL in the FRALLE and GRAALL 03-05 protocols. Comparison of mutational profiles by pathways between IKZF1 ${ }^{\text {Alt }}$ T-ALLs $(n=26)$ and IKZF1 GL T-ALLs ( $\left.n=450\right)$, with a focus on alterations found in at least $5 \%$ of the whole cohort. Percentage frequencies in each group are indicated. Genes are grouped by functional categories. ${ }^{*} p<0.05$. (D) Overall survival (OS) and (E) Cumulative Incidence of Relapse $(\mathrm{CIR})$ in FRALLE and GRAALL Treated patients. 


\begin{tabular}{|c|c|c|c|c|c|c|c|c|c|c|c|c|}
\hline & & & & No & (\%) of Patien & & & & & & & \\
\hline Characteristic & & IKZF1 Alt & & & $\mathrm{KZF}^{\mathrm{GL}}$ & & & Total & & & $p$ & \\
\hline Total & 26 & (5.5\%) & 45 & 50 & (94.5\% & & 476 & $(100$ & & & & \\
\hline Clinical subsets analyze & & & & & & & & & & & & \\
\hline Male sex & 21 & $(81 \%)$ & 33 & 39 & $(75 \%)$ & & 360 & $(75$ & & & 0.6 & \\
\hline Median age, years & 23.5 & {$[1.1 ; 59.1]$} & 15 & 5.2 & {$[1.2 ; 59$} & & 15.4 & {$[1.1 ; 5$} & 9.1 & & 0.1 & \\
\hline Median WBC (G/L) & 74.1 & {$[2.8 ; 641]$} & 63 & 3.4 & {$[0.3 ; 980$} & & 63.8 & {$[0.3 ;$} & 980] & & 0.3 & \\
\hline CNS involvement & $4 / 26$ & $(15 \%)$ & $47 /$ & 448 & $(11 \%)$ & & $51 / 474$ & $(11$ & & & 0.5 & \\
\hline ETP classification & $4 / 16$ & $(25 \%)$ & $51 /$ & 291 & $(18 \%)$ & & $55 / 307$ & $(18$ & & & 0.5 & \\
\hline T-cell receptor status & 17 & & 29 & 95 & & & 312 & & & & & \\
\hline Immature $(\mathrm{IMO} / \delta / \gamma)$ & 8 & $(47 \%)$ & 6 & 0 & $(20 \%)$ & & 68 & $(22$ & & & 0.02 & * \\
\hline Cortical (IMB, pre $\alpha \beta)$ & 5 & $(29 \%)$ & 15 & 54 & $(52 \%)$ & & 159 & $(51$ & & & 0.08 & \\
\hline Mature TCR $\alpha \beta$ & 3 & $(18 \%)$ & 4 & 2 & $(14 \%)$ & & 45 & $(14$ & & & 0.7 & \\
\hline Mature TCR $\delta \delta$ & 1 & $(6 \%)$ & 3 & 9 & $(13 \%)$ & & 40 & $(13$ & & & 0.7 & \\
\hline Oncogenetics & 24 & & 35 & 90 & & & 414 & & & & & \\
\hline$T L X 1$ & 1 & $(4 \%)$ & 5 & 3 & $(14 \%)$ & & 54 & $(13$ & & & 0.3 & \\
\hline$T L X 3$ & 4 & $(17 \%)$ & 6 & 8 & $(17 \%)$ & & 72 & $(17$ & & & 0.9 & \\
\hline SIL-TAL1 & 2 & $(8 \%)$ & 5 & 5 & $(14 \%)$ & & 57 & $(14$ & & & 0.6 & \\
\hline CALM-AF10 & 1 & $(4 \%)$ & 1 & 2 & $(3 \%)$ & & 13 & $(3 \%$ & & & 0.5 & \\
\hline None of above & 16 & $(67 \%)$ & 20 & 02 & $(52 \%)$ & & 218 & $(53$ & & & 0.2 & \\
\hline HOXA positive & $4 / 21$ & $(19 \%)$ & $74 /$ & 315 & $(23 \%)$ & & $78 / 336$ & $(24$ & & & 0.8 & \\
\hline$N / F-R / P$ classifier & 26 & & 45 & 50 & & & 476 & & & & & \\
\hline High Risk classifier & 15 & $(58 \%)$ & 19 & 98 & $(44 \%)$ & & 213 & $(45$ & & & 0.2 & \\
\hline NOTCH1/FBXW $7^{\text {mut }}$ & 19 & $(73 \%)$ & 30 & 02 & $(67 \%)$ & & 321 & $(67$ & & & 0.7 & \\
\hline$K / N-R A S^{m u t}$ & 8 & $(31 \%)$ & 4 & 1 & $(9 \%)$ & & 49 & $(10$ & & & 0.003 & * \\
\hline PTEN altered & 2 & $(8 \%)$ & 8 & 0 & (18\%) & & 82 & $(17$ & & & 0.3 & \\
\hline Treatment response & & & & & & & & & & & & \\
\hline $\mathrm{CR}$ & $24 / 26$ & (92\%) & $416 /$ & /450 & (92\%) & & $440 / 476$ & $(92$ & & & 1 & \\
\hline Prednisone response & $14 / 26$ & $(54 \%)$ & 245 & /441 & $(56 \%)$ & & $259 / 467$ & $(55$ & & & 1 & \\
\hline MRD1 $\geq 10^{-4}$ & $12 / 18$ & $(67 \%)$ & $111 /$ & /322 & $(34 \%)$ & & $123 / 340$ & $(36$ & & & 0.01 & * \\
\hline $\mathrm{HSCT}$ & $3 / 24$ & $(13 \%)$ & $93 /$ & 416 & $(22 \%)$ & & $96 / 440$ & $(22$ & & & 0.3 & \\
\hline Outcome & & & & & & & & & & & & \\
\hline 5-year CIR [95\% CI] & $50 \%$ & {$[32 ; 71]$} & & $28 \%$ & {$[24 ; 32]$} & & $29 \%$ & {$[25 ; 33]$} & & & 0.01 & * \\
\hline 5 -year OS $[95 \% \mathrm{Cl}]$ & $37 \%$ & {$[19 ; 55]$} & & $73 \%$ & {$[69 ; 77]$} & & $71 \%$ & {$[67 ; 75]$} & & & $<0.001$ & * \\
\hline & & & nivariate a & and $m$ & ultivariate a & nalysis & & & & & & \\
\hline & & & ivariate & & & & & Multiv & Iaria & & & \\
\hline CIR & SHR & & $95 \% \mathrm{Cl}$ & & $p$ & SHR & & $95 \% \mathrm{Cl}$ & & $p$ & & \\
\hline Age* & 1.01 & ( 0.98 & 1.03 & ) & 0.57 & - & - & - & & - & & \\
\hline $\log (W B C)^{*}$ & 1.62 & ( 1.20 & ; 2.18 & ) & 0.02 & 1.60 & ( 1.18 & ; 2.17 & 1 & 0.003 & & \\
\hline Prednisone response & 0.67 & ( 0.47 & ; 0.95 & ) & 0.026 & 0.92 & ( 0.63 & ; 1.34 & ) & 0.66 & & \\
\hline 4-gene classifier** & 2.78 & ( 1.94 & ; $\quad 3.99$ & ) & $<0.001$ & 2.69 & ( 1.86 & ; 3.88 & ) & $<0.001$ & & \\
\hline$I K Z F 1^{\text {alt }}$ & 2.12 & ( 1.17 & ; 3.86 & ) & 0.013 & 2.15 & ( 1.18 & ; 3.91 & 1 & 0.012 & & \\
\hline OS & $\mathrm{HR}$ & & $95 \% \mathrm{Cl}$ & & $p$ & $\mathrm{HR}$ & & $95 \% \mathrm{Cl}$ & & $p$ & & \\
\hline Age* & 1.03 & ( 1.01 & ; 1.05 & ) & 0.001 & 1.04 & ( 1.02 & ; 1.06 & ) & $<0.001$ & & \\
\hline $\log (\mathrm{WBC})^{*}$ & 1.99 & ( 1.48 & ; 2.67 & ) & $<0.001$ & 2.04 & ( 1.49 & ; 2.79 & ) & $<0.001$ & & \\
\hline Prednisone response & 0.54 & ( 0.38 & 0.76 & ) & $<0.001$ & 0.79 & ( 0.55 & ; 1.15 & ) & 0.21 & & \\
\hline 4-gene classifier** & 2.93 & ( 2.06 & 4.17 & ) & $<0.001$ & 2.88 & ( 2.00 & ; 4.16 & ) & $<0.001$ & & \\
\hline$I K Z F 1^{\text {alt }}$ & 2.94 & ( 1.74 & 4.96 & ) & $<0.001$ & 2.80 & ( 1.61 & 4.88 & ) & $<0.001$ & & \\
\hline
\end{tabular}

Table 1: Clinico-biological and outcome characteristics of adult and pediatric T-ALLs (GRAALL and FRALLE protocols) according to IKZF1 status. 
Figure 1.

IKZF1 Alterations

A IKZF1 Mutations $(\mathrm{n}=42 / 82)$

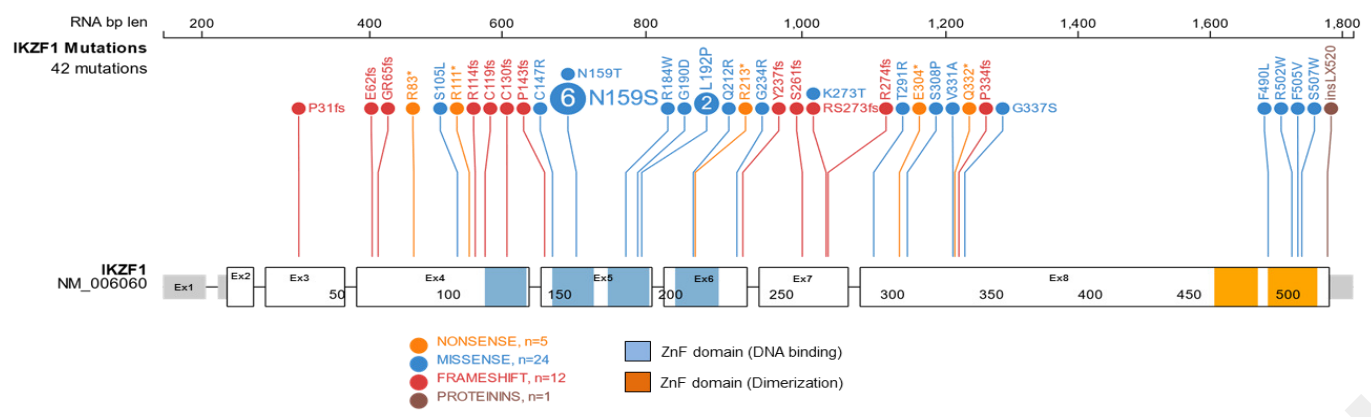

B IKZF1 Deletions $(n=40 / 82)$

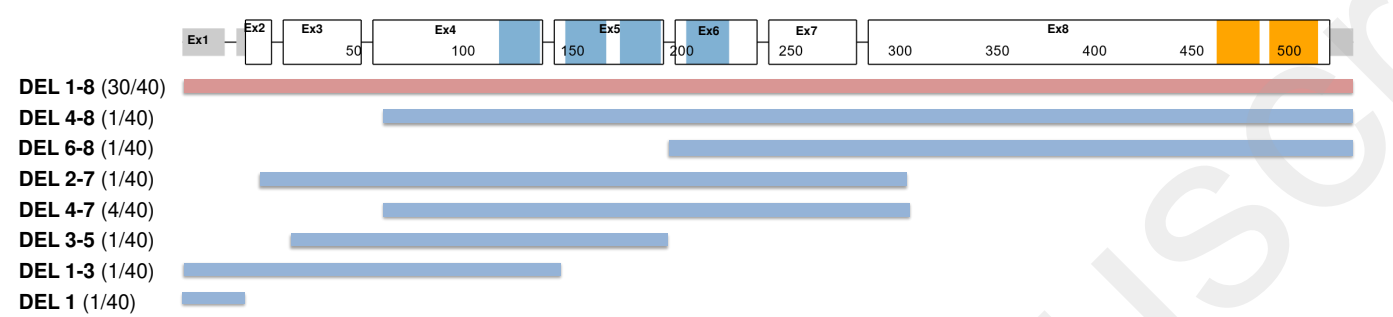

C

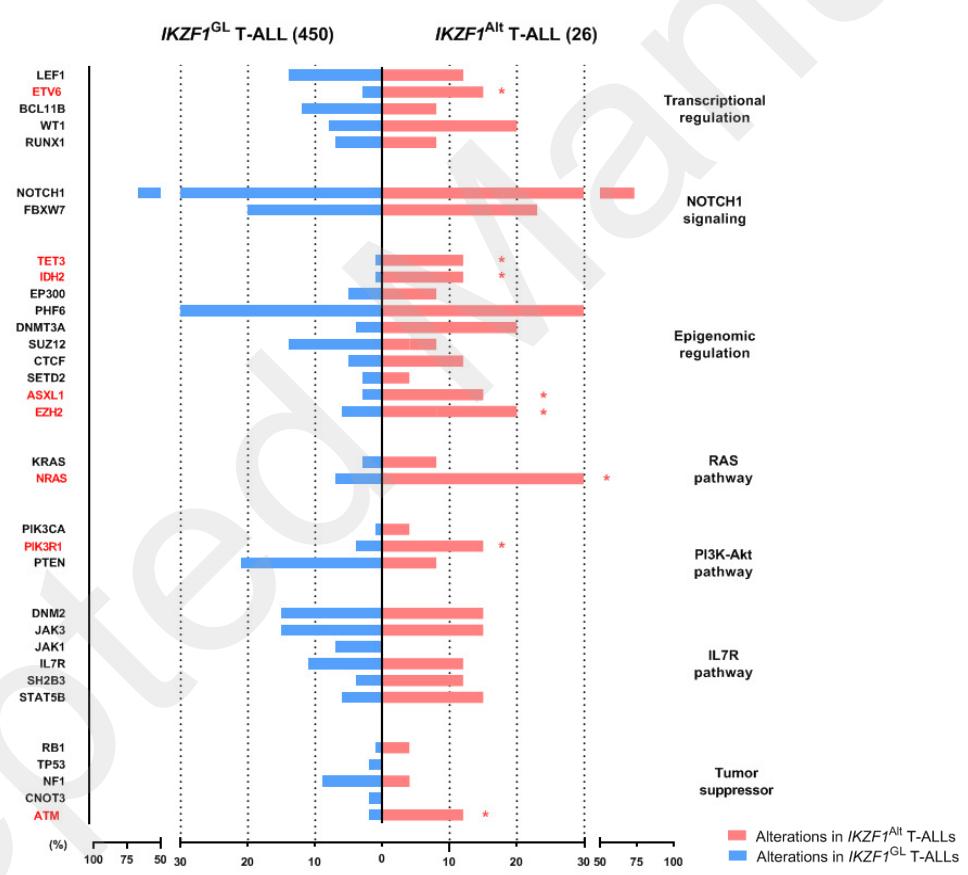

D

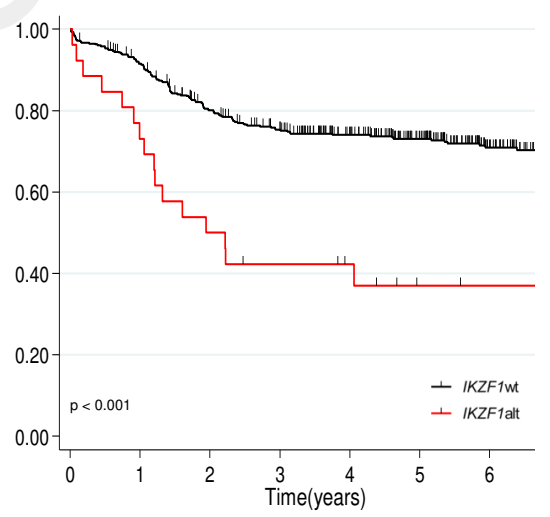

Number at risk

$\begin{array}{lcccccc}\text { IKZF1wt } 450 & 404 & 342 & 308 & 261 & 212 & 139 \\ \text { IKZF1alt } 26 & 19 & 13 & 10 & 8 & 4 & 3\end{array}$

E

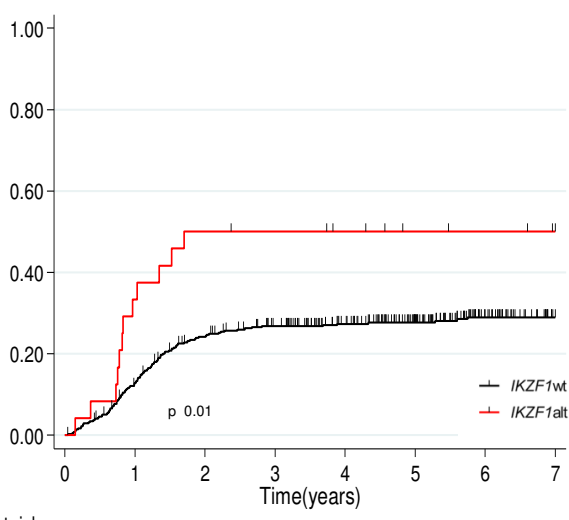

Number at risk

$\begin{array}{lccccccc}\text { IKZF1wt 416 } & 356 & 303 & 278 & 240 & 194 & 137 & 99\end{array}$

(D) Overall survival (OS) and (E) Cumulative Incidence of Relapse (CIR) in FRALLE and GRAALL treated patients. 